\title{
PROKLA-Redaktion
}

\section{Editorial: Religion, Politik und Ökonomie}

Huntington is back again! Vor mehr als zwei Jahrzehnten prägte Samuel Huntington - zunächst als Frage, dann als Behauptung - die Formel vom Clash of $C i$ vilizations, der dem Westen, nachdem ihm der Kommunismus der realsozialistischen Länder abhanden gekommen war, neue Feindbilder benannte. 1993 veröffentlichte er in der Zeitschrift Foreign Affairs den Aufsatz „The Clash of Civilizations?" und 1996 folgte die Monographie Clash of Civilizations and the Remaking of World Order, die zu einem internationalen Bestseller wurde. In einer deutschen Kurzfassung des Aufsatzes führte Huntington seine zentralen Gedanken aus: „Meine These ist, daß die grundlegende Ursache von Konflikten in dieser neuen Welt nicht ideologischer oder wirtschaftlicher Natur sein wird. Der wichtigste Grund für die Konflikte wird kulturell bestimmt sein. Nationalstaaten werden zwar die mächtigsten Akteure auf dem Globus bleiben, die grundsätzlichen Auseinandersetzungen der Weltpolitik aber werden zwischen Nationen und Gruppierungen aus unterschiedlichen Kulturen auftreten. Der Zusammenprall der Zivilisationen (civilizations) wird die Weltpolitik beherrschen ..." Diese Kulturen definierten sich „sowohl durch gemeinsame objektive Elemente - wie Sprache, Geschichte, Religion, Gebräuche, Institutionen - wie durch das subjektive Selbstverständnis der Menschen, die in ihr leben." Die Anzahl der "großen“ Kulturen, die weitgehend mit geografischen Räumen gleichgesetzt wurden, bezifferte Huntington mit ,sieben oder acht ... die westliche, die konfuzianische, die japanische, die islamische, die hinduistische, die slawisch-orthodoxe, die lateinamerikanische und möglicherweise die afrikanische Kultur." Zum Zusammenprall dieser unterschiedlichen Kulturen müsse es "nach dem Ende der ideologischen Spaltung Europas" kommen, denn nun tauche „die kulturelle Teilung Europas zwischen westlichem Christentum einerseits und orthodoxem Christentum und Islam andererseits wieder auf." Die „zentrale Achse der zukünftigen Weltpolitik" würde Huntington zufolge „aller Wahrscheinlichkeit nach, um den Ausdruck eines Politikers aus Singapur, Kishore Mahbubani, aufzugreifen, der Konflikt zwischen den 'Westen und dem Rest', also die Antwort nichtwestlicher Kulturen auf Macht und Werte des Westens sein." (Alle Zitate aus Huntington 1993)

Diese Anrufung gemeinsamer westlicher Werte passte gut in eine Zeit, in der in vielen Ländern ein mehr oder weniger starker Abbau sozialstaatlicher Einrichtungen und wirtschaftspolitischer Regulierungen auf der Tagesordnung stand. Statt drastisch zunehmende wirtschaftliche und soziale Ungleichheiten zu benennen, griffen maßgebliche PolitikerInnen und PublizistInnen zu dem rhetorischen Rettungsanker des ethnischen und religiösen „Wir“. Demnach ging es nicht um 
explodierende Manager-Boni oder die wachsende Konzentration von Vermögen bei den obersten fünf Prozent der Bevölkerung, denen immer mehr working poor und sonstige Arme gegenüberstanden, sondern um ganz andere Trennungslinien: um die „Fremden“ im eigenen Land, die sich angeblich allesamt in „Parallelgesellschaften" abschotteten. Gern wurde, insbesondere in Deutschland, die Rede von einer traditionellen „christlich-jüdischen Wertegemeinschaft" im Munde geführt und dabei ein bemerkenswert lückenhaftes Geschichtsverständnis offenbar, bei dem Jahrhunderte der antisemitischen Verfolgungen bis hin zum Holocaust einfach ausgeblendet wurden. So brachte etwa der bayerische Ministerpräsident Horst Seehofer (CSU) 2010 die „,hristlich-jüdische Leitkultur" gegen angebliche Islamisierung und kulturelle Überfremdung in Anschlag und goss damit Öl ins Feuer der seinerzeit anhaltenden Sarrazin-Debatte (siehe auch den Beitrag von Daniel Keil zu Pegida und zur Aktualisierung des Völkischen in PROKLA 180/2015).

Zwar entlarvten kritische Intellektuelle schnell die perfide Logik der Argumentation: „Zuerst beschwören Politiker eine Wertegemeinschaft, die religiös und kulturell so fugendicht geschlossen wird ('christlich-jüdisch'), dass Andersgläubige darin von vornherein keinen Platz finden können. In einem zweiten Schritt wird die homogene 'Wertegemeinschaft' dann so lange mit den bürgerlichen Grundrechten zu einer unauflöslichen Einheit verschmolzen, bis der elementare Unterschied zwischen Verfassungsnormen und kulturellen Werten unkenntlich wird“, so schrieb etwa Thomas Assheuer in Die Zeit und schloss sich damit einem Argument von Ulrich K. Preuß an (Assheuer 2010; kursiv durch die Verfasser). Doch für viele hatte sich die These vom Krieg gegen die
Werte „des Westens“vor allem mit den Anschlägen vom 11. September 2001 in New York bestätigt. Die US-Regierung hatte unmittelbar danach den War on Terror ausgerufen, und erklärte damals zwar ausdrücklich, es handele sich um einen Kampf gegen Al Qaida, also gegen "den Terror", nicht aber um den Krieg gegen eine Religion. Doch in der öffentlichen Diskussion wurde "der Terrorismus" immer häufiger mit "dem Islam" als solchem gleichgesetzt. Huntington selbst sprach sich im Übrigen dagegen aus, die Attacke auf das World Trade Center als Kulturkampf zu interpretieren und warnte eindringlich vor dem von der Regierung George W. Bush ab 2003 losgetretenen Irakkrieg (siehe auch den Beitrag von Robert Brenner in PROKLA 149/2007). Was bis dahin vor allem als Trennungslinie innerhalb der nationalen Gesellschaften diagnostiziert worden war, wiederholte sich nun also auf der internationalen Ebene: „Wir“ gegen „die Anderen“. Und „die Anderen“ wurden immer mehr mit AnhängerInnen des Islam gleichgesetzt.

Allerdings stießen Huntingtons ursprüngliche Thesen inzwischen auch auf weitverbreitete Kritik: Sein Verständnis von Kulturen, Zivilisationen und Räumen sei eher beliebig und die Begriffe seien zum Teil austauschbar bzw. unklar voneinander abgegrenzt; er unterstelle dabei Blöcke, die in sich homogen und weitestgehend unveränderbar gedacht sind, insofern sei sein Ansatz ahistorisch und essentialistisch. Wegweisend sind in diesem Zusammenhang bis heute die Arbeiten zu Okzidentalismus und Neorassismus von Theoretikern wie Edward Said, Stuart Hall und Étienne Balibar.

Eine Untersuchung der BertelsmannStiftung von $2006 \mathrm{kam}$ zu dem Schluss, in 119 untersuchten Ländern könne man keinen „Kampf der Kulturen“ feststellen, 
vielmehr seien in den letzten Jahren die wesentlichen Ursachen für politische Gewalt „Armut, ethnische Spaltung, Staatsschwäche, internationale Faktoren und Mängel des politischen Systems" (Croissant/ Hartmann 2006). Selbst die FAZ sprach in einem Artikel anlässlich Huntingtons Tod im Jahr 2008 vom „Primat des Politischen“ und monierte, es ließe sich „in jedem soziologischen Proseminar, in jeder Eckkneipe lernen, dass Konflikte in den seltensten Fällen genuin ethnischer oder religiöser Natur sind, sondern eine soziale Motivation haben. Der Mensch lebt eben nicht in Werten allein, als hingen sie lose an einem Ideenhimmel.“ (Geyer 2008)

Huntingtons apokalyptische Thesen in der Nachfolge von Oswald Spengler und Arnold Toynbee schienen über die Jahre somit bei einem großen Teil der Publizistik an Strahlkraft eingebüßt zu haben. Doch dies änderte sich spätestens nach den $\mathrm{Pa}$ riser Attentaten gegen die Redaktion von Charlie Hebdo und einen jüdischen Supermarkt im Januar 2015. Es war der frühere Präsident Nicolas Sarkozy, der Huntingtons Slogan vom „Kampf der Kulturen" wieder aufnahm, wofür er von französischen SozialistInnen damals noch geschmäht wurde - kurz darauf bediente sich Premierminister Manuel Valls jedoch genau dieser Terminologie. Wurde unmittelbar nach den Attentaten ein vorgeblicher "Charlie-Geist" der Versöhnlichkeit, Brüderlichkeit und des republikanischen Zusammenstehens hochgehalten, so erklärte Valls im Juni 2015 in einem RadioInterview: „Es handelt sich hier nicht um einen Krieg zwischen dem Okzident und dem Islam, sondern um einen Krieg der Werte, die die unsrigen sind und die wir mit anderen auch außerhalb von Europa teilen.“ Dieser Krieg finde „auch im Herzen des Islam statt, und zwar zwischen der einen Seite, die humanistische Werte vertritt, und der anderen, dem obskurantischen und totalitäten Islam, der uns seine Vorstellung von Gesellschaft aufzwingen will“ (Biseau 2015; Übersetzung: D.S.). Doch die Unterscheidung zwischen dem humanistischen und dem obskurantistischen Islam blieb letzten Endes nicht mehr als ein Lippenbekenntnis und in der politischen Praxis folgenlos. Denn die französische Regierung führt tatsächlich Krieg, im Äußeren seit September 2015 mit Luftangriffen gegen den sogenannten Islamischen Staat (IS) in Syrien, im Inneren mit polizeilichen Mitteln gegen die Jugendlichen in den Banlieues (siehe dazu auch den Beitrag von Martin Kronauer in PROKLA 149/2007).

Wenn die jugendlichen Attentäter, meist Franzosen mit migrantischen Wurzeln, ihre Attacken mit dem Ruf „Allahu Akbar" begleiten, werden sie stets als islamistisch etikettiert, doch haben SozialwissenschaftlerInnen wiederholt darauf hingewiesen, wie wenig sie mit dieser Religion überhaupt zu tun haben. Der Politologe Olivier Roy beschreibt sie als Angehörige einer Generation, die angesichts ihrer langjährigen Marginalisierung und Perspektivlosigkeit in der französischen Gesellschaft revoltieren - eine Revolte um der Revolte willen. Sie hätten keinerlei politische Ziele, ihre Kenntnis des Islam sei rudimentär, ihre Verbindung zur islamischen Gemeinschaft äußerst lose, und sie hätten sich kaum jemals an deren Riten beteiligt. Ihr Ziel sei die einmalige Selbstermächtigung, die ihnen der Salafismus anbietet: „Sie töten so wie die Massenmörder in Amerika oder Breivik in Norwegen, kalt und ruhig. Der Nihilismus und der Hochmut sind eng miteinander verbunden ... Es geht hier nicht um die 
Radikalisierung des Islam, sondern um die Islamisierung der Radikalität." (Roy 2015; Übersetzung: D.S.) Ähnlich lautet die Diagnose von Dominique Moïsi in einem Interview: „Mehrere Studien zeigen, dass die jungen Jihadisten den Islam kaum kennen; sie interessiert einzig die Gewalt. Sie sind näher bei Clockwork Orange als beim Islam." (Der Standard, 7.1.2016)

Unbeantwortet bleibt freilich in beiden Interpretationen die Frage, warum die JihadistInnen in Frankreich und anderswo nicht sozialrevolutionär auftreten oder warum sich ganz allgemein GlaubenskriegerInnen aller Weltreligionen überhaupt religiös radikalisieren, anstatt politisch das Ziel einer grundlegenden Veränderung der gesellschaftlichen Verhältnisse zu verfolgen, unter denen sie entwürdigt und geknechtet werden. Warum wollen sie selbst knechten, warum sind sie bereit, anstatt Solidarität mit den Schwachen zu praktizieren, beliebige und ihnen unbekannte Menschen zu quälen und zu ermorden, Frauen zu drangsalieren, Akte kultureller Barbarei zu praktizieren und sich den Wissensfortschritten zu verschließen? Dies hat auch mit der Schwäche der Linken in Frankreich und in anderen Ländern zu tun.

Derweil setzt die französische Regierung ihre bereits unter Sarkozy eingeschlagene Linie des „Kärcherns“ fort, also der desolaten Situation der Jugendlichen in den Vorstädten nach wie vor einzig mit Repression zu begegnen und die wirtschaftlichen und gesellschaftlichen Hintergründe ihrer extremen Entfremdung und Ausgrenzung zu verleugnen. Auch in Deutschland fanden sich beredte Wiedergängerinnen Huntingtons wie Necla Kelek und Seyran Ateş, die durch Talkshows und Feuilletons tourten und dort nach wie vor als vorgeblich besonders authentische Expertinnen gern gesehen werden. Zwangsverheiratungen, Ehrenmorde oder gewalttätige Jugendliche sind für sie allgemeine und typische Merkmale „des“Islam, was ihnen die Frage nach den gesellschaftlichen Hintergründen derartiger Exzesse erspart, genauso wenig wie sie Debatten und Aktivitäten innerhalb der islamischen Community, die solche Verhaltensweisen deutlich verurteilt und gegen sie angeht, zur Kenntnis nehmen. Wie Birgit Rommelspacher zeigt, hat die Popularität des Kulturalismus praktische Konsequenzen. Forschungen zum Rechtsextremismus konzentrieren sich stets auf deutsche Jugendliche und deren soziales Umfeld, während türkische Jugendliche, die etwa im Dunstkreis der faschistischen Grauen Wölfe agieren, einzig im Zusammenhang mit ihrer Religion gesehen werden. Daraus folgt: „Für rechtsextreme deutsche Jugendliche werden psychosoziale Beratungskonzepte entwickelt und pädagogische Jugendprogramme ausgelegt, während in Bezug auf die Radikalisierung muslimischer Jugendlicher die Bekämpfung des Islam oder gar die Ausweisung der Jugendlichen aus Deutschland gefordert wird." (Rommelspacher 2009: 445)

Es hat von anderer Seite zahlreiche Bemühungen gegeben, zwischen verschiedenen Spielarten des Islam zu differenzieren, und selbst die 2007 veröffentlichte Studie des Bundesinnenministeriums Muslime in Deutschland unterscheidet zwischen orthodoxen, fundamental orientierten, radikalen und säkularen Richtungen. Im Übrigen kommt die Studie auch zu dem Ergebnis, 94 Prozent der befragten Muslime lehnten Gewalt ab, außerdem gebe es keinen Zusammenhang zwischen dem Grad der Religiosität und der Befürwortungvon Gewalt (Rommelspacher 2009: 443, 446). Dennoch hat das Bild einer durchweg Gewalt legitimierenden Religion massiv an Boden gewonnen. Was zunächst ein plattes Erklärungsmuster von RechtspopulistInnen zu sein schien, findet nun auch bei 
Intellektuellen, die sich als AufklärerInnen verstehen, zunehmend Anklang. Der „Kampf der Kulturen“ trägt seither immer stärker religiöse Züge. Wenn der Ägypter Hamed Abdel Samad wortreich vertritt, Islam und Faschismus seien von Grund auf wesensverwandt (2014), stößt diese These in vielen Medien und in einer breiten Öffentlichkeit auf große Resonanz, da sie eine einfache Erklärung für das Vordringen und die mörderischen Praktiken des IS im Nordirak anbietet. Als arabisch-deutscher Intellektueller scheint Samad dabei über den Verdacht des antimuslimischen Rassismus erhaben und wurde trotz - oder gerade wegen? - seiner islamkritischen Positionen im Jahr 2011 von Bundesinnenminister Thomas de Maizière als Teilnehmer in die 2. Deutsche Islamkonferenz berufen. Wie viele andere AutorInnen auch geht er darüber hinweg, dass etwa der Begriff Jihad innerhalb der islamischen Welt durchaus unterschiedlich und widersprüchlich interpretiert wird - die Bandbreite reicht von der legitimen Gewalt gegen Ungläubige bis zum individuellen inneren Kampf um den richtigen Glauben. Dazu kommt, dass häufig mit zweierlei Maß gemessen wird. Die Brutalitäten des sunnitisch orientierten IS gelten westlichen PolitikerInnen als islamistisch, während die seit Jahrzehnten ähnlich menschenverachtende Politik der am Wahhabismus orientierten saudiarabischen Regierung nur vorübergehend und fallweise Aufsehen erregt und Kritik herausfordert. Neben Peitschenhieben und Amputationen gehört eine wachsende Zahl von Todesurteilen zum Strafrepertoire des Regimes. So wurden nach Recherchen von Amnesty International 2015 bis zum November nicht weniger als 152 Menschen hingerichtet, meist durch Kreuzigung oder öffentliche Enthauptung, und Anfang Januar 2016 gab es an einem einzigen Tag 47 Tötungen. Doch das Land gilt der westlichen Politik als „Stabilitätsanker“ in der Region, wird daher seit Jahrzehnten hofiert und stetig mit Waffen, unter anderem aus den USA und Deutschland, beliefert.

Auf internationaler Ebene ist die Vorstellung, es gebe einen einzigen, in sich homogenen Islam, mindestens ebenso irrig wie auf der nationalen Ebene, zerfällt er doch in eine kaum zu übersehende Zahl von Richtungen und Gruppierungen. In vielen Ländern tolerieren sich diese gegenseitig und pflegen auch mit anderen, etwa christlichen Gemeinschaften gute und enge Kontakte. Tatsächlich berufen sich alle monotheistischen Religionen auf den gleichen Gott, und das Gebot der Nächstenliebe ist ihnen allesamt eingeschrieben. In seiner Rede bei der Verleihung des Friedenspreises des deutschen Buchhandels im Oktober 2015 hat Navid Kermani am Beispiel des katholischen Klosters Mar Musa in Syrien eindrucksvoll beschrieben, wie daraus gesellschaftliche Praxis werden kann. In diesem Kloster trafen sich von jeher nicht nur Christen aus aller Welt, sondern es wurden selbstverständlich auch Muslime eingeladen und aufgenommen, ob sie in Not waren oder nicht. Als Pater Jacques, der dem Kloster vorstand, zusammen mit anderen Mitgliedern des Klosters von IS-Kämpfern entführt wurde, waren es Muslime aus den Dörfern der Nachbarschaft, die sich unter Einsatz ihres Lebens für seine Befreiung einsetzten, die ihnen schließlich auch gelang (Kermani 2015). Das Beispiel steht für eine Vielzahl historischer und gegenwärtiger Erfahrungen, dass Religionen mit verschiedenen Werthorizonten sich keineswegs immer bekämpfen müssen, sondern relativ friedlich koexistieren können. Dies war etwa der Fall in 
Andalusien zwischen dem 8. und dem 14. Jahrhundert, als ChristInnen, Juden und Jüdinnen sowie MuslimInnen mit- und nebeneinander lebten und arbeiteten, selbst wenn die Religionen keineswegs in allem gleichberechtigt waren. In heutiger Zeit gibt es ebenfalls Beispiele derartiger Koexistenzen, etwa von Juden und Jüdinnen sowie MuslimInnen in Marokko oder von Buddhismus und Shintoismus in Japan. Am Beginn des 20. Jahrhunderts wurde die bis heute lebendige ökumenische Bewegung für die Zusammenarbeit christlicher Kirchen ins Leben gerufen, und in Berlin ist seit 2014 das Projekt House of One im Entstehen: ein Bet- und Lehrhaus, unter dessen gemeinsamem Dach sich eine Kirche, eine Synagoge und eine Moschee befinden sollen.

Dennoch zeigt die Geschichte auch, dass nahezu jede Religion extrem autoritäre Züge und Alleinvertretungsansprüche entwickeln kann, jede von ihnen vor dem Hintergrund sozialer und politischer Spannungen ein „Wir" anrufen kann, das es gegen die einer anderen Religion Angehörenden zu verteidigen gilt, und zwar mit allen zu Gebote stehenden Mitteln. In seinem Roman Judas lässt $\mathrm{Amos} \mathrm{Oz}$ einen pensionierten Jerusalemer Gymnasialleiter die entsprechenden historischen Erfahrungen zugespitzt so formulieren: „Das Judentum und das Christentum und auch der Islam predigen den Nektar des Mitleids nur so lange, wie sie selbst über keine Fesseln, keine Macht, keine Folterkeller und keine Galgen verfügen.“ (Oz 2015: 77)

Wenn es zu Glaubenskriegen zwischen KatholikInnen und ProtestantInnen kam, ging es jedoch nie allein um Religion, sondern stets auch um politische Interessen, so etwa Ende des 16. Jahrhunderts beim Aufstand der protestantischen Fürsten unter Führung von Moritz von Sachsen gegen den katholischen Kaiser Karl V, bei dem die Fürsten für ihre Freiheit und der Kaiser für seine unangefochtene Macht kämpften. Ähnlich verhält es sich mit den jahrzehntelangen blutigen Auseinandersetzungen in der zweiten Hälfte des 20. Jahrhunderts in Nordirland, bei denen auf der einen Seite diejenigen standen, die bäuerlicher Herkunft, relativ arm und katholisch waren, auf der anderen Seite diejenigen, die von der Industrie lebten, relativ wohlsituiert und protestantisch waren.

Das Ineinandergreifen von politischen und religiösen Motiven ist also alles andere als neu, die Beliebtheit des Kulturparadigmas führt derzeit jedoch dazu, dass gerade, wenn es um den Islam geht, gesellschaftliche Bedingungen weitgehend ausgeblendet werden und die Religion als treibendes Motiv in den Vordergrund gestellt wird so auch bei den aktuellen äußerst heftigen Konflikten innerhalb der muslimischen Welt, wie bei den Konfrontationen zwischen dem Iran und Saudi-Arabien, innerhalb des Iraks oder im Jemen. In seinem Beitrag untersucht Sascha Radl am Beispiel des Jemen daher die politökonomischen Hintergründe für den Aufstieg von Ansar Allah („Houthis“), wie es also dazu kam, dass diese schiitisch-zaidistische Bewegung sich gegen die sunnitische Zentralregierung durchsetzen konnte, was schließlich auch zum militärischen Eingreifen SaudiArabiens führte. Ein weiterer Beitrag, bei dem der Islam im Mittelpunkt steht, beleuchtet diesen als Staatsreligion im Iran, wobei Katharina Götsch der Frage nachgeht, welche Bedeutung dem religiösen Moment (insbesondere in der sunnitisch-schiitischen Auseinandersetzung innerhalb der islamischen Mächte) in den Außenbeziehungen des Iran zukommt. Dieser trat programmatisch als Schutzmacht der Schiiten auf und schrieb sich den Export der islamischen Revolution auf die Fahnen, doch wurde diese Orientierung in 
der praktischen Politik immer wieder von profanen Interessen und pragmatischen Erwägungen durchkreuzt.

Das Verhältnis von Religion und Moderne ist aber auch in ganz anderen gesellschaftlichen Konstellationen zu thematisieren. Lange Zeit wurde im Anschluss an Max Webers Diktum von der „Entzauberung der Welt" erwartet, dass Religiosität und die Bedeutung kirchlicher Institutionen mit wirtschaftlichem Wachstum, Industrialismus und Massenkonsum an Bedeutung verlieren würden: die „Säkularisierung" sei eine Entwicklung, die mit der Durchsetzung der Moderne notwendigerweise einhergehe. Dieser Befund entsprang immer schon einer europäisch-ethnozentrischen Haltung und traf etwa für die USA, aber auch für andere außereuropäische Länder stets nur begrenzt zu. So ist in den letzten Jahrzehnten gerade die Durchsetzung neoliberaler Wirtschaftskonzepte in vielen Fällen damit verbunden, dass Regierungen deren gesellschaftlich desintegrierende Wirkungen mit der verstärkten Förderung religiöser Institutionen abfedern und kompensieren oder ihre eigene Autorität mit derjenigen der Kirche zusätzlich festigen wollen. Beispiele dafür finden sich in der jüngeren Geschichte in unterschiedlichsten Weltregionen: So betreibt etwa die Partei für Gerechtigkeit und Entwicklung AKP in der Türkei eine verstärkte Islamisierung des Landes und verfolgt zugleich eine zum Staatsprojekt erhobene Strategie der Neoliberalisierung; damit ähnelt sie verblüffend der streng katholischen und radikal wirtschaftsliberalen Partei PAN in Mexiko. Auch in früheren Ländern des Ostblocks bemühen sich autoritäre Regierungen um eine zunehmende Allianz von Staat und Kirche, so etwa in Russland
Präsident Putin um diejenige mit der russisch-orthodoxen Kirche, wobei das „neue Russland" traditionelle Werte in einer zunehmend fragmentierten Gesellschaft durchsetzen soll und "Gotteslästerung" (wie im Fall feministischen Aktivistinnen von Pussy Riot) als politische Allzweckwaffe dienen kann. Ähnlich betont der polnische Präsident Jaroslaw Kaczyński bei jeder sich bietenden Gelegenheit, die katholische Kirche gehöre zu den wichtigsten Fundamenten des Polentums, und donnert: "Jede Hand, die sich gegen die Kirche erhebt, ist eine Hand, die sich gegen Polen erhebt.“" (Pollack 2016) Auf einer anderen Ebene, aber mit ähnlichen politischen Intentionen der Stabilisierung extrem autoritärer Systeme, ist die Offensive evangelischer Sekten in den Ländern Mittelamerikas seit den 1980er Jahren zu sehen, als sie die militärische Aufstandsbekämpfung auf ideologischer Ebene flankierten, um den politisch unbequemen, befreiungstheologischen Gemeinden das Wasser abzugraben - wobei sowohl die eine als auch die andere Strategie massive Unterstützung aus den USA erfuhr. Einen aktuellen Fall der Verbindung neoliberaler Wirtschaftspolitik mit religiösem Fundamentalismus untersucht Andreas Wiebel für Indien, wo Premierminister Narendra Modi seit seinem Wahlerfolg 2014 den Versuch gestartet hat, die Öffnung des Landes für internationales Kapital durch einen nationalidentitären Hinduismus zu begleiten - ein Vorhaben, das manche Beobachter auch mit dem Begriff „Hindufaschismus“ charakterisieren.

Doch Religion ist keineswegs nur Mittel für die Durchsetzung von Herrschaft und autoritärer Politik, sondern sie ist oftmals ein möglicher sozialer Identitäts- und 
Hoffnungsanker für Deklassierte und Depravierte, wenn nicht sogar Ausgangspunkt von Mobilisierungen für ein besseres Leben und eine bessere Gesellschaft. Die Bedeutung von Religion erfährt also möglicherweise nicht nur „,on oben“, als Instrument von Regierungen oder politischen Gruppen, eine Renaissance, sondern auch an der Basis wird von den Sozialwissenschaften eine „Wiederkehr der Religiosität" diagnostiziert. Sie konnte für die „Mühseligen und Beladenen“ (Matthäusevangelium) oder für diejenigen, die im „wirklichen Elend“ (Marx) lebten, immer schon Trost und Hoffnung bieten, soziale Identität sowie die Zugehörigkeit zu einer Gemeinschaft bedeuten und - wie etwa die Befreiungstheologie in Lateinamerika - sogar ein Versprechen der Emanzipation bieten, somit nicht nur ein Mittel der Unterwerfung, sondern genauso ein positives Angebot darstellen. Damit bildet die (vermeintlich) neue Entdeckung der Religiosität, ob in ihrer sozialrevolutionären oder in ihrer reaktionären Variante, gerade auch für 'die Linke' eine besondere Herausforderung. Denn in ein 'klassisches' Rechts-Links-Schema lassen sich religiöse Bewegungen oft nur schwer einordnen.

In den ersten Jahrzehnten nach dem Zweiten Weltkrieg trat der Kapitalismus in vielen europäischen Ländern und auch in den USA in einer wohlfahrtsstaatlichen Variante auf, die ein Glücks- und Wohlstandsversprechen enthielt, das sich für einige Jahrzehnte zu erfüllen schien, anderswo (etwa in mehreren afrikanischen Ländern) ging die Modernisierung mit staatssozialistischen Experimenten einher, die ähnliche Hoffnungen transportierten. Die meisten dieser Entwicklungen waren fürs Erste mit wachsender religiöser Gleichgültigkeit verbunden. Der spätere Abbau sozialstaatlicher Regelungen bewirkte demgegenüber stets ein krasses
Anwachsen materieller Unterschiede und soziale Polarisierung, sodass große Gruppen von VerliererInnen und Enttäuschten entstanden, was vielfach einen Nährboden für die Popularität religiöser Bewegungen schuf, die häufig nicht nur spirituelle Orientierung, sondern auch praktische Hilfen anbieten. In diesem Zusammenhang untersucht Ingar Solty den Aufschwung evangelikaler Bewegungen in den USA, wo insbesondere die Entstehung von „Megakirchen“ ein herausragendes Phänomen der letzten Jahrzehnte darstellt. Im neoliberalen Workfare-Staat (der vom rechten Protestantismus vehement befürwortet wird), machen sie Angebote für die Freizeitgestaltung, erlauben das Knüpfen von Freundschaften und Arbeitsnetzwerken oder sorgen für Altenpflege und Kinderbetreuung. Demgegenüber stellt sich Stephan Lanz in seinem Beitrag gegen die These, neue Religiosität verdanke sich stets in erster Linie der zunehmenden Verbreitung von Armut und Ausgrenzung. Diese Annahme widerspreche der Realität von Metropolen, wie anhand von Fallstudien für Istanbul, Lagos, Rio de Janeiro und Berlin gezeigt werden kann. Hier stoße man auf „Prosperitätsreligionen“, die in christlichen oder muslimischen Varianten auftreten, von jungen, gebildeten und kosmopolitischen Milieus getragen werden und sozialen Aufstieg mit Werten wie Askese, Gemeinschaftlichkeit, Missionierung und persönlicher Verantwortlichkeit verbinden.

Zusätzlich zu all diesen Beiträgen, die sich mit unterschiedlichsten Formen neuer Religiosität befassen, greift Andreas Arndt mit der Frage „Kapitalismus als Religion?" ein Thema auf, das bereits vor mehr als zwei Jahrzehnten den Gegenstand eines PROKLA-Artikels darstellte (das Heft 96/1994 hatte den Schwerpunkt Fundamentalismus und neue Religiosität). Wie Andreas Arndt 
ging Rudolf Thiessen (1994) damals von Walter Benjamins Textfragment aus, in dem dieser Anfang der 1920er Jahre die These vertrat, der Kapitalismus sei eine reine Kultreligion, ohne Dogmatik und ohne Theologie. Die Diskussion um Benjamins provokativen Text ist mittlerweile vielfach weiterentwickelt worden. Andreas Arndt stellt sie kurz vor und setzt sie fort, indem er fragt, inwieweit Benjamins Überlegungen tatsächlich in Beziehung zu marxistischen Vorstellungen von Kapitalismus stehen, oder nicht eher zu literarischen Vorlagen, in denen Antikapitalismus mit apokalyptischen Erwartungen verbunden wird.

In jedem Fall, so unser Plädoyer, ist Religion heute als Bestandteil moderner nationalstaatlich-kapitalistischer Vergesellschaftung zu analysieren. Nicht zuletzt gilt es dabei, animistischen oder spiritualistischen Anschauungen, wie sie zum Teil auch Bestandteil monotheistischer Religionen sind, ein wissenschaftlich-theoretisches Weltverständnis entgegenzusetzen - und zwar nicht im Sinne empiristischer und positivistischer Erklärungen, sondern im Sinne einer rationalen Reflexionsfähigkeit und eines emanzipatorischen politischen Projekts. Das ist das beste Antidot gegen religiös begründete Ideologien wie auch gegen die kulturalisierende und essentialistische These vom Clash of Civilizations, wie sie von Samuel Huntingtons 'Nachahmungstätern' (heute wieder) vertreten wird.

Außerhalb des Schwerpunkts diskutiert Sarah Bormann das Phänomen, dass auf dem Feld der Arbeitsbeziehungen seit einigen Jahren Non-Governmental Organizations (NGOs) an Bedeutung gewinnen, zum Beispiel in der ITK-Industrie. In ihrem Artikel diskutiert sie die Kampagnenstrategien von NGOs und stellt die Frage, welche Chancen aber auch Grenzen öffentlichkeitswirksame Protesttaktiken von NGOs haben und was dies für die Bildung von Bündnissen zwischen Gewerkschaften und NGOs bedeutet.

In der letzten PROKLA diskutierte Dario Azzelini vor dem Hintergrund der jüngeren Entwicklungen in Griechenland und Spanien die Potenziale linker Parteipolitik, einen transformativen Prozess innerhalb der bestehenden Institutionen voranzutreiben. Syriza stehe für ein Scheitern dieser Strategie, so Azzelini kritisch. Die Partei habe es versäumt, sich stärker auf eine gesellschaftliche und institutionell möglicherweise nicht mehr kanalisierbare Protestdynamik einzulassen. Auch Podemos drohe, sich von den sozialen Bewegungen abzukoppeln, um für eine Mehrheit wählbar zu sein. Mario Candeias widerspricht in der vorliegenden PROKLA der Einschätzung. Azzelini habe ein zu enges Verständnis von Partei und man müsse stärker darüber nachdenken, wie das Spannungsverhältnis zwischen Partei und Bewegungen - zwischen Kooperation und Konflikt - politisch gestaltet werden kann.

Mit der erfolgreichen Erpressung der griechischen Syriza-Regierung machten die Herrschenden in Europa deutlich, dass sie bereit sind, ein Abweichen von ihrer Krisenpolitik im Zweifel mit allen Mitteln zu unterbinden. Im Anschluss an dieses aggressive Vorgehen hat Michael Heinrich in PROKLA 180 die These vertreten, dass diese Austeritätspolitik nicht einer „Fehlkonstruktion" des Euro geschuldet ist, die sich letztlich auch gegen das deutsche Exportmodell wenden muss, wie häufig angenommen, sondern gewollt und im Interesse Deutschlands sei - ,gute deutsche Politik" eben. Mit ihrer empirischen Studie in PROKLA 181 haben Frederic 
Heine und Thomas Sablowski diese These untermauert: Deutschland zieht nicht nur Kapital aus der Peripherie der Eurozone ab, sondern verlagert auch die Absatzmärkte seiner Exportindustrie immer stärker von der Eurozone weg auf den Weltmarkt und profitiert dabei vom schwachen Euro. Doch hat sich Deutschland in der Eurokrise damit auch zu jener unangefochtenen Führungsmacht in Europa aufgeschwungen, die die EU immer vorgab zu verhindern? Und fällt der deutschen Linken damit umgekehrt auch eine Schlüsselrolle für emanzipatorische Politik in ganz Europa zu? Dieser These widerspricht Peter Wabl mit seinem Einspruch in dieser PROKLA: Entgegen einer auch in der Linken weitverbreiteten Annahme sei „das Narrativ der deutschen Dominanz oder gar Hegemonie in der EU völlig überzogen“.

Aber wie sollte es die Linke dann mit der EU halten? Nicht erst seit der „Eurokrise" und der deutschen Dominanzpolitik gegenüber der Syriza-Regierung treibt diese Frage zu Recht viele um, polarisiert und drängt aber auch andere wichtige Fragen in den Hintergrund - allen voran jene, wer der politische und soziale Träger grundlegender Veränderung sein kann. Zusammen mit der jour fixe initiative Berlin lädt die PROKLA dazu ein, am 13. März in der Neuen Gesellschaft für bildende Kunst (NGBK) mit Stefanie Hürtgen und Peter Wablüber diese Fragen zu diskutieren. Wir werden die Debatte in den kommenden Ausgaben weiterführen .

\section{Henrik Lebubn und Dorothea Schmidt} (für die Redaktion)

\section{Literatur}

Assheuer, Thomas (2010): Die neuen Feinde. Das Gerede von der 'christlich-jüdischen Leitkultur' schürt den Fremdenhass. In: Die Zeit, Nr. 43, 21.10.2010. URL: zeit. de/2010/43/Leitkultur,Zugriff: 11.12.2015.

Biseau, Grégoire: „Guerre de civilisation“: quand Valls contredit Valls. URL: liberation.fr/societe/2015/06/28/guerre-des-civilisationsquand-valls-contredit-valls_1338931, Zugriff: 8.1.2016.

Croissant, Aurel/Hartmann Hauke (2006): Der Kampf der Kulturen findet kaum statt. In. Frankfurter Rundschau, 21.11.2006. URL: fr-online.de/doku---debatte/der-kampfder-kulturen-findet-kaum-statt, 1472608, 2879810.html, Zugriff: 8.1.2016.

Geyer, Christian (2008): Der Ohrwurm. URL: faz.net/aktuell/feuilleton/zum-tod-von-samuel-p-huntington-der-ohrwurm-1745797. html, Zugriff: 8.1.2016.

Huntington, Samuel (1993): Im Kampf der Kulturen.URL_http://www.zeit.de/1993/33/ im-kampf-der-kulturen, Zugriff: 8.1.2016.

Interview mit Dominique Moïsi (2016). In: Der Standard: „Eher Clockwork Orange als Koran“, 7.1.2016.

Kermani, Navid (2015): Jacques Mourad und die Liebe in Syrien. URL: faz.net/aktuell/ feuilleton/debatten/kermanis-friedenspreis-rede-jacques-mourad-und-die-liebein-syrien-13863150.html, Zugriff: 8.1.2016.

Oz, Amos (2015): Judas, Berlin.

Pollack, Martin (2016): Ist „mein“ Polen schon verloren? In: Der Standard, 9.1.2016.

Rommelspacher, Birgit (2009): Islamkritik und antimuslimische Positionen am Beispiel von Necla Kelek und Seyran Ateş. In: Schneiders, Thorsten Gerald (Hg.): Islamfeindlichkeit: Wenn die Grenzen der Kritik verschwinden, Wiesbaden: 433-455.

Roy, Olivier (2015): Le djihadisme est une révolte générationelle et nihiliste. In: $L e$ Monde, 24.11.2015.

Samad, Hamed Abdel (2014): Der islamische Faschismus, München.

Thiessen, Rudolf(1994): Kapitalismus als Religion. In: PROKLA 24(3): 400-418. 